PROCEEDINGS OF THE

AMERICAN MATHEMATICAL SOCIETY

Volume 134, Number 4, Pages 973-981

S 0002-9939(05)08244-4

Article electronically published on September 28, 2005

\title{
ON COMPLEX AND NONCOMMUTATIVE TORI
}

\author{
IGOR NIKOLAEV
}

(Communicated by Michael Stillman)

\begin{abstract}
The "noncommutative geometry" of complex algebraic curves is studied. As a first step, we clarify a morphism between elliptic curves, or complex tori, and $C^{*}$-algebras $T_{\theta}=\left\{u, v \mid v u=e^{2 \pi i \theta} u v\right\}$, or noncommutative tori. The main result says that under the morphism, isomorphic elliptic curves map to the Morita equivalent noncommutative tori. Our approach is based on the rigidity of the length spectra of Riemann surfaces.
\end{abstract}

\section{INTRODUCTION}

Noncommutative geometry is a branch of algebraic geometry studying "varieties" over noncommutative rings. The noncommutative rings are usually taken to be rings of operators acting on a Hilbert space [7]. The rudiments of noncommutative geometry can be traced back to F. Klein [3], 4] or even earlier. The fundamental modern treatise [1] gives an account of status and perspective of the subject.

The noncommutative torus $T_{\theta}$ is a $C^{*}$-algebra generated by linear operators $u$ and $v$ on the Hilbert space $L^{2}\left(S^{1}\right)$ subject to the commutation relation $v u=$ $e^{2 \pi i \theta} u v, \theta \in \mathbb{R}-\mathbb{Q}[11$. The classification of noncommutative tori was given in [2], 8], 11]. Recall that two such tori $T_{\theta}, T_{\theta^{\prime}}$ are Morita equivalent if and only if $\theta, \theta^{\prime}$ lie in the same orbit of the action of group $G L(2, \mathbb{Z})$ on irrational numbers by linear fractional transformations.

It is remarkable that the "moduli problem" for $T_{\theta}$ looks as such for the complex tori $E_{\tau}=\mathbb{C} /(\mathbb{Z}+\tau \mathbb{Z})$, where $\tau$ is complex modulus. Namely, complex tori $E_{\tau}, E_{\tau^{\prime}}$ are isomorphic if and only if $\tau, \tau^{\prime}$ lie in the same orbit of the action of $S L(2, \mathbb{Z})$ on complex numbers by linear fractional transformations. It was observed by some authors (e.g. [5], 15]) that it might not be just a coincidence. This note is an attempt to show that it is indeed so: there exists a general morphism between Riemann surfaces and $C^{*}$-algebras.

Let us give rough idea of our approach. Given Riemann surface $S$, there is a function $S \rightarrow \mathbb{R}_{+}^{\infty}$ which maps the (discrete) set of closed geodesics of $S$ to a discrete subset of a real line by assigning each closed geodesic its riemannian length. If $T_{g}(S)$ is the space of all Riemann surfaces of genus $g \geq 0$, then the function

$$
\mathfrak{W}: T_{g}(S) \longrightarrow \mathbb{R}_{+}^{\infty}
$$

Received by the editors February 25, 2003 and, in revised form, November 2, 2004.

2000 Mathematics Subject Classification. Primary 14H52, 46L85.

Key words and phrases. Elliptic curve, noncommutative torus.

(C)2005 American Mathematical Society 
is finite-to-one and "generically" one-to-one [17]. In the case $g=1$, function $\mathfrak{W}$ is always one-to-one. It is known also that restriction $\mathfrak{W}_{\text {syst }}: T_{g}(S) \rightarrow \mathbb{R}_{+}$of $\mathfrak{W}$ to the shortest closed geodesic of $S$ (called systole) is a $C^{0}$ Morse function on $T_{g}(S)$ [13, $\S 5$. Below we focus on the case $g=1$, i.e. $T_{1} \simeq E_{\tau}$.

Recall that $T_{\theta}$ has a unique state $s_{0}$ (which is actually a tracial state) [11. Any positive functional on $T_{\theta}$ has form $\omega s_{0}$, where $\omega>0$ is a real number. Let $\Theta=\left\{T_{\theta} \mid \theta \in \mathbb{R}-\mathbb{Q}\right\}$ and $\Omega=\{\omega \in \mathbb{R} \mid \omega>0\}$. We define a map

$$
\mathfrak{V}: \Theta \times \Omega \longrightarrow \mathbb{R}_{+}^{\infty}
$$

by the formula $\left(T_{\theta}, \omega\right) \mapsto\left\{f_{n}(\omega) \ln \operatorname{tr}\left(A_{n}\right)\right\}_{n=0}^{\infty}$, where

$$
\begin{aligned}
A_{0} & =\left(\begin{array}{cc}
a_{0} & 1 \\
1 & 0
\end{array}\right), \\
A_{1} & =\left(\begin{array}{cc}
a_{0} & 1 \\
1 & 0
\end{array}\right),\left(\begin{array}{cc}
a_{1} & 1 \\
1 & 0
\end{array}\right), \\
& \vdots \\
A_{n} & =\left(\begin{array}{cc}
a_{0} & 1 \\
1 & 0
\end{array}\right)\left(\begin{array}{cc}
a_{1} & 1 \\
1 & 0
\end{array}\right) \ldots\left(\begin{array}{cc}
a_{n} & 1 \\
1 & 0
\end{array}\right)
\end{aligned}
$$

are integer matrices whose entries $a_{i}>0$ are partial denominators of the continued fraction expansion of $\theta$, and $f_{n}$ are monotone $C^{0}$ functions of $\omega$. Assuming that functions $\mathfrak{W}, \mathfrak{V}$ have common range, one gets a mapping $\mathfrak{W V}^{-1}: E_{\tau} \rightarrow\left(T_{\theta}, \omega\right)$.

Morphisms between $E_{\tau}$ and $T_{\theta}$ have been studied in [5], 9], 10], 16]. The works [5], 10] and [16] treat noncommutative tori as "quantum compactification" of the space of elliptic curves. This approach deals with an algebraic side of the subject. In particular, Manin [5] suggested to use "pseudolattices" (i.e. $K_{0}$-group of $T_{\theta}$ ) to solve the multiplication problem for real number fields. This problem is part of the Hilbert 12th problem. In [9] a functor from derived category of holomorphic vector bundles over $T_{\theta}$ to the Fukaya category of such bundles over $E_{\tau}$ was constructed. In this note we prove the following results.

Theorem 1. Let $E_{\tau}$ be a complex torus of modulus $\tau, \operatorname{Im} \tau>0$, and let $\left(T_{\theta}, \omega\right)$ be a pair consisting of noncommutative torus with an irrational Rieffel's parameter $\theta$ and a positive functional $T_{\theta} \rightarrow \mathbb{C}$ of norm $\omega$. Then there exists a one-to-one mapping $E_{\tau} \rightarrow\left(T_{\theta}, \omega\right)$. The action of the modular group $S L(2, \mathbb{Z})$ on the complex half plane $\{\tau \in \mathbb{C} \mid$ Im $\tau>0\}$ is equivariant with:

(i) the action of group $G L(2, \mathbb{Z})$ on irrationals $\{\theta \in \mathbb{R}-\mathbb{Q} \mid \theta>0\}$ by linear fractional transformations;

(ii) a discrete action on positive reals $\{\omega \in \mathbb{R} \mid \omega>0\}$.

In particular, isomorphic complex tori map to the Morita equivalent noncommutative tori, and vice versa.

Definition 1. The irrational number $\theta$ of mapping $E_{\tau} \rightarrow\left(T_{\theta}, \omega\right)$ we call a projective curvature of the elliptic curve $E_{\tau}$.

Theorem 2. Projective curvature of an elliptic curve with complex multiplication is a quadratic irrationality.

\section{Proofs}

The proof of both theorems is based on the rigidity of length spectrum of complex torus; cf. Wolpert [17. Preliminary information on complex and noncommutative tori can be found in Section 2. 
1.1. Proof of Theorem 1. Let us review the main steps of the proof. By the rigidity lemma (Lemma 11) the length spectrum $S p E$ defines conformal structure of $E$. In fact, this correspondence is a bijection. Under isomorphisms of $E$ the length spectrum can acquire a real multiple or get a "cut of finite tail" (Lemma 21). We attach to $\mathbb{C} / L$ a continued fraction of its projective curvature $\theta$ as specified in the Introduction. Then isomorphic tori $\mathbb{C} / L$ will have continued fractions which differ only in a finite number of terms. In other words, one can attach a Morita equivalence class of noncommutative tori to every isomorphism class of complex tori.

Lemma 1 (Rigidity of length spectrum). Let $S p E$ be the length spectrum of a complex torus $E=\mathbb{C} / L$. Then there exists a unique complex torus with the spectrum. This correspondence is a bijection.

Proof. See McKean [6].

Let $S p X=\left\{l_{1}, l_{2}, \ldots\right\}$ be the length spectrum of a Riemann surface $X$. Let $a>$ 0 be a real number. By $a S p X$ we understand the length spectrum $\left\{a l_{1}, a l_{2}, \ldots\right\}$. Similarly, for any $m \in \mathbb{N}$ we denote by $S p_{m} X$ the length spectrum $\left\{l_{m}, l_{m+1}, \ldots\right\}$, i.e. the one obtained by deleting the first $(m-1)$-geodesics in $S p X$.

Lemma 2. Let $E \sim E^{\prime}$ be isomorphic complex tori. Then either:

(i) $S p E^{\prime}=|\alpha| S p E$ for an $\alpha \in \mathbb{C}^{\times}$, or

(ii) $S p E^{\prime}=S p_{m} E$ for a $m \in \mathbb{N}$.

Proof. (i) The complex tori $E=\mathbb{C} / L, E^{\prime}=\mathbb{C} / M$ are isomorphic if and only if $M=\alpha L$ for a complex number $\alpha \in \mathbb{C}^{\times}$. It is not hard to see that the closed geodesic of $E$ are bijective with the points of the lattice $L=\omega_{1} \mathbb{Z}+\omega_{2} \mathbb{Z}$ in the following way. Take a segment of a straight line through points 0 and $\omega$ of lattice $L$ which contains no other points of $L$. This segment represents a homotopy class of curves through 0 and a closed geodesic of $E$. Evidently, this geodesic will be the shortest in its homotopy class with the length $|\omega|$ equal to an absolute value of the complex number $\omega$. Thus, $|\omega|$ belongs to the length spectrum of $E$.

Now let $S p E=\left\{\left|\omega_{1}\right|,\left|\omega_{2}\right|, \ldots\right\}$ with $\omega_{i} \in L$. Since $M=\alpha L$, one gets $S p E^{\prime}=$ $\left\{|\alpha|\left|\omega_{1}\right|,|\alpha|\left|\omega_{2}\right|, \ldots\right\}$ and $S p E^{\prime}=|\alpha| S p E$. Item (i) follows.

(ii) Note that according to (i) the length spectrum $S p X=\left\{l_{0}, l_{1}, l_{2}, \ldots\right\}$ can be written as $S p X=\left\{1, l_{1}, l_{2}, \ldots\right\}$ after multiplication on $1 / l_{0}$, where $l_{0}$ is the length of the shortest geodesic. Note also that the shortest geodesic of the complex torus has a homotopy type $(1,0)$ or $(0,1)$ (standard generators for $\pi_{1} E$ ).

Let $a, b, c, d$ be integers such that $a d-b c= \pm 1$ and let

$$
\begin{aligned}
& \omega_{1}^{\prime}=a \omega_{1}+b \omega_{2}, \\
& \omega_{2}^{\prime}=c \omega_{1}+d \omega_{2}
\end{aligned}
$$

be an automorphism of the lattice $L=\omega_{1} \mathbb{Z}+\omega_{2} \mathbb{Z}$. This automorphism maps standard generators $(1,0)$ and $(0,1)$ of $L$ to the vectors $\omega_{1}=(a, b), \omega_{2}=(c, d)$. Let their lengths be $l_{m}, l_{m+1}$, respectively.

As we showed earlier, $l_{m}, l_{m+1} \in S p E$, and it is not hard to see that there are no geodesics of the intermediate length. (This gives a justification for the notation chosen.) Note that $\omega_{1}, \omega_{2}$ are standard generators for the complex torus $E^{\prime} \sim E$, and therefore one of them is the shortest closed geodesics of $E^{\prime}$. One can normalize it to the length 1 . 
On the other hand, there are only a finite number of closed geodesics of length smaller than $l_{n}$ (McKean [6]). Thus $S p E \cap S p E^{\prime}=\left\{l_{m}, l_{m+1}, \ldots\right\}$ for a finite number $m$ and since (4) is automorphism of the lattice $L$. In other words, $S p E^{\prime}=$ $S p_{m} E$. Item (ii) follows.

To finish the proof of item (i) of Theorem 1, one needs to combine Lemmas 1 and 2 with the fact that two noncommutative tori $T_{\theta}, T_{\theta^{\prime}}$ are Morita equivalent if and only if their continued fractions differ only in a finite number of terms (Section $2.1)$.

To prove item (ii) of Theorem 1, let to the contrary the action of $S L(2, \mathbb{Z})$ be nondiscrete, i.e. having limit points in $\Omega$. Let $p=\lim _{n \rightarrow \infty}\left(T_{\theta_{n}}, \omega_{n}\right)$, where $\theta_{n}$ lie in the same orbit of $G L(2, \mathbb{Z})$. Let $E_{p}$ be the corresponding complex torus such that $E_{p} \neq E_{p_{n}}$ are nonisomorphic. By continuity of the systole function $\mathfrak{W}_{\text {syst }}$ (see the Introduction), $S p E_{p}=\lim S p E_{p_{n}}$. Then by the rigidity of length spectra, $E_{p} \cong E_{p_{n}}$ are isomorphic. The contradiction proves item (ii) of the theorem.

1.2. Proof of Theorem 2. Let us outline the idea of the proof. If $E$ admits complex multiplication, then its complex modulus $\tau$ lies in an imaginary quadratic field $K$. In fact, up to an isogeny, the ring of endomorphisms End $E=\mathcal{O}_{K}$, where $\mathcal{O}_{K}$ is the ring of integers of field $K$. It can be shown that $L$ is an ideal in $\mathcal{O}_{K}$ (Section 2.3). The length spectrum $S p E$ of an elliptic curve with complex multiplication is a "geometric progression" with the growth rate $|\alpha|$, where $\alpha \in E n d E$ (Lemma 3). One can use Klein's lemma (Lemma 4) to characterize length spectra in terms of continued fractions. In particular, length spectrum with asymptotically geometric growth correspond to periodic continued fractions. Thus, projective curvature converges to quadratic irrationality.

Definition 2. Length spectrum $S p E$ of an elliptic curve $E=\mathbb{C} / L$ is called $\alpha$ multiplicative, if there exists a complex number $\alpha \in \mathbb{C}^{\times}$with $|\alpha|>1$ such that

$$
S p E=\left\{l_{1}, \ldots, l_{N},|\alpha| l_{1}, \ldots,|\alpha| l_{N}, \ldots,|\alpha|^{n} l_{1}, \ldots,|\alpha|^{n} l_{N}, \ldots\right\},
$$

for $N \in \mathbb{N}$.

Lemma 3. Let $E$ be an elliptic curve with complex multiplication. Then its length spectrum $S p E$ is $\alpha$-multiplicative for an $\alpha \in \mathbb{C}^{\times}$.

Proof. Let $E=\mathbb{C} / L$ be a complex torus which admits nontrivial endomorphisms $z \mapsto \alpha z, \alpha \in K=\mathbb{Q}(\sqrt{-d})$. It is known that End $E$ is an order in the field $K$. In fact, up to an isogeny of $E, E n d E \simeq \mathcal{O}_{K}$, where $\mathcal{O}_{K}$ is the ring of integers of imaginary quadratic field $K$ (Section 2.3). Lattice $L$ in this case corresponds to an ideal in $\mathcal{O}_{K}$.

Let $l_{1}$ be the minimal length of a closed geodesic of $E$. For an endomorphism $\alpha: E \rightarrow E, \alpha \in \mathbb{C}^{\times}$, consider the set of geodesics whose lengths are less than $|\alpha| l_{1}$. By the properties of $S p E$ mentioned in Section 1.1, such a set will be finite. Let us denote the lengths of geodesics in this set by $l_{1}, \ldots, l_{N}$. Since every geodesic in $S p E$ is a complex number $\omega_{i}$ lying in the ring $L \subseteq \mathcal{O}_{K}$, one can consider the set of geodesics $\alpha \omega_{1}, \ldots, \alpha \omega_{N}$. The length of these geodesics will be $|\alpha| l_{1}, \ldots,|\alpha| l_{N}$, respectively. It is not hard to see that by the choice of number $N$, the first $2 N$ elements of $S p E$ are presented by the following growing sequence of geodesics: $l_{1}, \ldots, l_{N},|\alpha| l_{1}, \ldots,|\alpha| l_{N}$. We proceed by iterations of $\alpha$, until all closed geodesics of $E$ are exhausted. The conclusion of Lemma 3 follows. 
We shall need the following statement regarding geometry of the regular continued fractions [3], 4]. It is valid for any regular fraction, not necessarily periodic.

Lemma 4 (F. Klein). Let

$$
\omega=\mu_{1}+\frac{1}{\mu_{2}+\frac{1}{\mu_{3}+\ldots}}
$$

be a regular continued fraction. Let us denote the convergents of $\omega$ by

$$
\frac{p_{-1}}{q_{-1}}=\frac{0}{1}, \quad \frac{p_{0}}{q_{0}}=\frac{1}{0}, \quad \frac{p_{1}}{q_{1}}=\frac{\mu_{1}}{1}, \ldots, \frac{p_{\nu}}{q_{\nu}}=\frac{\mu_{\nu} p_{\nu-1}+p_{\nu-2}}{\mu_{\nu} q_{\nu-1}+q_{\nu-2}} .
$$

For any lattice $L$ in $\mathbb{C}$, consider a segment $I$ with ends in the points $\left(p_{\nu-2}, q_{\nu-2}\right)$ and $\left(p_{\nu}, q_{\nu}\right)$. Then the segment $J$ which joins 0 with the point $p_{\nu-1}, q_{\nu-1}$ is parallel to $I$ and

$$
|I|=\mu_{\nu}|J|,
$$

where $|\bullet|$ denotes the length of the segment.

Proof. We refer the reader to [4].

Corollary 1. Let $\omega_{\nu}=\left(p_{\nu}, q_{\nu}\right)$ be lattice points mentioned in Lemma 4. Then the length of vector $\omega_{\nu}$ can be evaluated with the help of the following asymptotic formula:

$$
\left|\omega_{\nu}\right| \approx\left|\omega_{\nu-2}\right|+\mu_{\nu}\left|\omega_{\nu-1}\right|
$$

Proof. Indeed, using the notation of Lemma 4, one can write $\left|\left(p_{\nu}, q_{\nu}\right)\right| \approx$ $\left|\left(p_{\nu-2}, q_{\nu-2}\right)\right|+|I|$. But according to equation (8) $),|I|=\mu_{\nu}\left|\left(p_{\nu-1}, q_{\nu-1}\right)\right|$. Corollary 1 follows.

Note that according to the recurrent formula (9) the length spectrum $\left\{\left|\omega_{\nu}\right|\right\}$ coming from continued fraction (6) is completely determined by the first two values: $\left|\omega_{1}\right|$ and $\left|\omega_{2}\right|$. Using (9), one can easily deduce the following asymptotic formula for $\left|\omega_{\nu}\right|$ as a function of $\left|\omega_{1}\right|,\left|\omega_{2}\right|$ :

$$
\left|\omega_{\nu}\right| \approx\left|\omega_{2}\right| \prod_{k=3}^{\nu} \mu_{k}+\left|\omega_{1}\right| \prod_{k=4}^{\nu} \mu_{k}+O(\nu)
$$

Fix $N$ a positive integer. It follows from equation (10) that

$$
\begin{aligned}
\lim _{\nu \rightarrow \infty} \frac{\left|\omega_{\nu+N}\right|}{\left|\omega_{\nu}\right|} & =\mu_{\nu+1} \ldots \mu_{\nu+N} \lim _{\nu \rightarrow \infty}\left(\frac{\mu_{\nu} \ldots \mu_{3}\left|\omega_{2}\right|+\mu_{\nu} \ldots \mu_{4}\left|\omega_{1}\right|+O(\nu)}{\mu_{\nu} \ldots \mu_{3}\left|\omega_{2}\right|+\mu_{\nu} \ldots \mu_{4}\left|\omega_{1}\right|+O(\nu)}\right) \\
& =\mu_{\nu+1} \ldots \mu_{\nu+N} .
\end{aligned}
$$

Let $E$ be an elliptic curve with complex multiplication. Then by Lemma 3 its length spectrum $S p E$ is $\alpha$-multiplicative. In other words,

$$
\frac{l_{\nu+N}}{l_{\nu}}=|\alpha|=\text { Const }
$$

for an $N \in \mathbb{N}$ and any $\nu \bmod N$. Note that $|\alpha|$ is a rational integer. Thus, by formula (11) we have $\mu_{\nu+1} \ldots \mu_{\nu+N}=$ Const, for any $\nu \bmod N$. The last requirement can be satisfied if and only if continued fraction (6) is $N$-periodic. Theorem 2 is proven. 


\section{BACKGROUND INFORMATION}

In the present section we briefly review noncommutative and complex tori. The excellent source of information on noncommutative torus are papers [2, [11] and a monograph of [12. The literature on complex torus is fairly vast. We recommend for the reference Ch. VI of [14].

2.1. Noncommutative torus. By the $C^{*}$-algebra one understands a noncommutative Banach algebra with an involution [12. Namely, a $C^{*}$-algebra $A$ is an algebra over $\mathbb{C}$ with a norm $a \mapsto\|a\|$ and an involution $a \mapsto a^{*}, a \in A$, such that $A$ is complete with respect to the norm, and such that $\|a b\| \leq\|a\|\|b\|$ and $\left\|a^{*} a\right\|=\|a\|^{2}$ for every $a, b \in A$. If $A$ is commutative, then the Gelfand theorem says that $A$ is isometrically $*$-isomorphic to the $C^{*}$-algebra $C_{0}(X)$ of continuous complex-valued functions on a locally compact Hausdorff space $X$. For otherwise, $A$ represents a "noncommutative" topological space $X$.

$K_{0}$ and dimension groups. Given a $C^{*}$-algebra, $A$, consider a new $C^{*}$-algebra $M_{n}(A)$, i.e. the matrix algebra over $A$. There exists a remarkable semi-group, $A^{+}$, connected to the set of projections in algebra $M_{\infty}=\bigcup_{n=1}^{\infty} M_{n}(A)$. Namely, projections $p, q \in M_{\infty}(A)$ are Murray-von Neumann equivalent $p \sim q$ if they can be presented as $p=v^{*} v$ and $q=v v^{*}$ for an element $v \in M_{\infty}(A)$. The equivalence class of projections is denoted by $[p]$. The semi-group $A^{+}$is defined to be the set of all equivalence classes of projections in $M_{\infty}(A)$ with the binary operation $[p]+[q]=[p \oplus q]$. The Grothendieck completion of $A^{+}$to an abelian group is called a $K_{0}$-group of $A$. The functor $A \rightarrow K_{0}(A)$ maps the unital $C^{*}$-algebras into the category of abelian groups so that the semi-group $A^{+} \subset A$ corresponds to a "positive cone" $K_{0}^{+} \subset K_{0}(A)$ and the unit element $1 \in A$ corresponds to the "order unit" [1] $\in K_{0}(A)$. The ordered abelian group $\left(K_{0}, K_{0}^{+},[1]\right)$ with the order unit is called a dimension (Elliott) group of $A$. The dimension (Elliott) group is a complete invariant of the $A F C^{*}$-algebras.

Noncommutative torus. Fix $\theta$ irrational and consider a linear flow $\dot{x}=\theta, \dot{y}=1$ on the torus. Let $S^{1}$ be a closed transversal to our flow. The noncommutative torus $T_{\theta}$ is a norm-closed $C^{*}$-algebra generated by the unitary operators in the Hilbert space $L^{2}\left(S^{1}\right)$ :

$$
U f(t)=z(t) f(t), \quad V f(t)=f(t-\alpha),
$$

which are multiplication by a unimodular function $z(t)$ and rotation operators. It could be easily verified that $U V=e^{2 \pi i \alpha} V U$. As an "abstract" algebra, $T_{\theta}$ is a crossed product $C^{*}$-algebra $C\left(S^{1}\right) \rtimes_{\phi} \mathbb{Z}$ of a (commutative) $C^{*}$-algebra of complexvalued continuous functions on $S^{1}$ by the action of powers of $\phi$, where $\phi$ is a rotation of $S^{1}$ through the angle $2 \pi \alpha$. $T_{\theta}$ is not $A F$, but can be embedded into an $A F$ algebra whose dimension group is $P_{\theta}$ (to be specified below); the latter is known to be intimately connected with the arithmetic of the irrational numbers $\theta$ 's. The following beautiful result is due to the efforts of many mathematicians 1 (Effros, Elliott, Pimsner, Rieffel, Shen, Voiculescu, etc.).

\footnotetext{
${ }^{1}$ The author apologizes for possible erroneous credits regarding the history of the problem. Classification of noncommutative tori seems to be an old problem; early results in this direction can be found in the works of Klein [3], 4].
} 
Theorem 3 (Classification of noncommutative tori). Let $T_{\theta}$ be a noncommutative torus. Suppose that the $\theta$ has a continued fraction expansion

$$
\theta=a_{0}+\frac{1}{a_{1}+\frac{1}{a_{2}+\ldots}} \stackrel{\text { def }}{=}\left[a_{0}, a_{1}, a_{2}, \ldots\right] .
$$

Let $\varphi_{n}$ be a composition of isometries of the lattice $\mathbb{Z}^{2} \subset \mathbb{R}^{2}: \quad \varphi_{n}=\left(\begin{array}{cc}a_{0} & 1 \\ 1 & 0\end{array}\right)$ $\ldots\left(\begin{array}{cc}a_{n} & 1 \\ 1 & 0\end{array}\right)$. Then $T_{\theta}$ can be embedded into an AF-algebra whose dimension group is a direct limit of the ordered abelian groups: $P_{\theta}=\lim _{n \rightarrow \infty}\left(\mathbb{Z}^{2}, \varphi_{n}\right)$. Moreover, if $\theta=\left[a_{0}, a_{1}, \ldots\right]$ and $\theta^{\prime}=\left[b_{0}, b_{1}, \ldots\right]$ are two irrational numbers, then $P_{\theta}$ and $P_{\theta^{\prime}}$ are isomorphic (i.e. noncommutative tori $T_{\theta}$ and $T_{\theta^{\prime}}$ are Morita equivalent) if and only if $a_{m+k}=b_{m}$ for an integer number $k \in \mathbb{Z}$. In other words, the irrational numbers $\theta$ and $\theta^{\prime}$ are modular equivalent: $\theta^{\prime}=\frac{a \theta+b}{c \theta+d}, \quad a d-b c= \pm 1$, where $a, b, c, d \in \mathbb{Z}$ are integer numbers.

Proof. An algebraic proof of this fact can be found in 2].

2.2. Complex torus. Let $L$ denote a lattice in the complex plane $\mathbb{C}$. Attached to $L$, there are the following classic Weierstrass function $\wp(z ; L)$ and Eisenstein series $G_{k}(L)$ :

$$
\begin{aligned}
\wp(z ; L) & =\frac{1}{z^{2}}+\sum_{\omega \in L^{\times}}\left\{\frac{1}{(z+\omega)^{2}}-\frac{1}{\omega^{2}}\right\}, \\
G_{k}(L) & =\sum_{\omega \in L^{\times}}^{k \geq 2} \frac{1}{\omega^{2 k}} .
\end{aligned}
$$

$\wp(z ; L)$ is analytic, and $G_{k}(L)$ is convergent for any lattice $L$ [14]. There exists a duality between lattices $L$ and cubic curves $E$ given by the following theorem.

Theorem 4. Let $L$ be a lattice in $\mathbb{C}$. Then the map $z \mapsto\left(\wp(z ; L), \frac{1}{2} \wp^{\prime}(z ; L)\right)$ is an analytic isomorphism from complex torus $\mathbb{C} / L$ to elliptic cubic $E=E(\mathbb{C})$ :

$$
E(\mathbb{C})=\left\{(x, y) \in \mathbb{C}^{2} \mid y^{2}=x^{3}-15 G_{4}(L) x-35 G_{6}(L)\right\} .
$$

Conversely, to any cubic in the Weierstrass normal form $y^{2}=x^{3}+a x+b$ there corresponds a unique lattice $L$ such that $a=-15 G_{4}(L)$ and $b=-35 G_{6}(L)$.

Proof. We refer the reader to 14 for a detailed proof of this fact.

Isomorphism of complex tori. Let $L$ be a lattice in $\mathbb{C}$. The Riemann surface $\mathbb{C} / L$ is called a complex torus. Let $f: \mathbb{C} / L \rightarrow \mathbb{C} / M$ be a holomorphic and invertible map (isomorphism) between two complex tori. Since $f$ is covered by a linear map $z \rightarrow \alpha z$ on $\mathbb{C}$, one can easily conclude that $\alpha L=M$ for an $\alpha \in \mathbb{C}^{\times}$. On the other hand, lattice $L$ can always be written as $L=\omega_{1} \mathbb{Z}+\omega_{2} \mathbb{Z}$, where $\omega_{1}, \omega_{2} \in \mathbb{C}^{\times}$and $\omega_{2} \neq k \omega_{1}$ for a $k \in \mathbb{R}$. The complex number $\tau=\frac{\omega_{2}}{\omega_{1}}$ is called a complex modulus of lattice $L$.

Lemma 5. Two complex tori are isomorphic if and only if their complex moduli $\tau$ and $\tau^{\prime}$ satisfy the equation

$$
\tau^{\prime}=\frac{a \tau+b}{c \tau+d} \quad a d-b c= \pm 1, \quad a, b, c, d \in \mathbb{Z} .
$$

Proof. The proof of this fact can be found in [14. 
2.3. Elliptic curves with complex multiplication. Let $E=\mathbb{C} / L$ be an elliptic curve. Consider the set End $E$ of analytic self-mappings of $E$. Each $f \in$ End $E$ is covered on the complex plane by map $z \mapsto \alpha z$ for an $\alpha \in \mathbb{C}$. It is not hard to see that End $E$ has the structure of a ring under the pointwise addition and multiplication of functions. The set End $E$ is called an endomorphism ring of an elliptic curve $E$. By the remarks above, End $E$ can be thought of as a subring of complex numbers:

$$
\text { End } E=\{\alpha \in \mathbb{C} \mid \alpha L \subset L\} .
$$

There exists a fairly complete algebraic description of such rings. Roughly speaking, they are either "rational integers" $\mathbb{Z}$ or integers $\mathcal{O}_{K}$ of an algebraic number field $K$. The following lemma is true.

Lemma 6. Let $\alpha \in$ End $E$ be a complex number. Then either:

(i) $\alpha$ is a rational integer, or

(ii) $\alpha$ is an algebraic integer in an imaginary quadratic number field $K=\mathbb{Q}(\sqrt{-d})$.

Proof. See 14 .

Complex multiplication. If End $E$ is different from $\mathbb{Z}, E$ is said to be an elliptic curve with complex multiplication. If $E$ admits complex multiplication, then its ring End $E$ is an order in an imaginary quadratic field $K$. In fact, $E$ admits an isogeny (analytic homomorphism) to a curve $E^{\prime}$ such that End $E^{\prime} \simeq \mathcal{O}_{K}$, where $\mathcal{O}_{K}$ is the ring of integers of field $K[14$. Thus, by property $\alpha L \subseteq L$, lattice $L$ is an ideal in $\mathcal{O}_{K}$. Denote by $h_{K}$ the class number of field $K$. It is well known that there exist $h_{K}$ nonisomorphic ideals in $\mathcal{O}_{K}$. Therefore, elliptic curves $E_{1}=\mathbb{C} / L_{1}, \ldots, E_{h_{K}}=$ $\mathbb{C} / L_{h_{K}}$ are pairwise nonisomorphic, but their endomorphism ring is the same [14.

\section{ACKNOWLEDGEMENTS}

It is my pleasure to thank G. A. Elliott, Yu. I. Manin and M. Rieffel for their interest in the subject of present note. I am grateful to the referee for his critical remarks and helpful suggestions.

\section{REFERENCES}

[1] A. Connes, Noncommutative Geometry, Academic Press, 1994. MR1303779 (95j:46063)

[2] E. G. Effros and C. L. Shen, Approximately finite $C^{*}$-algebras and continued fractions, Indiana Univ. Math. J. 29 (1980), 191-204. MR0563206 (81g:46076)

[3] F. Klein, Über eine geometrische Auffassung der gewöhnlichen Kettenbruchentwicklung, Nachrichten der Kgl. Gesellschaft der Wissenschaften zu Göttingen, Math.-phys. Klasse (1895), Heft 3.

[4] F. Klein, Ausgewählte Kapitel der Zahlentheorie, Math. Annalen 48 (1896/97).

[5] Yu. I. Manin, Real multiplication and noncommutative geometry, The legacy of Niels Henrik Abel, Springer (2004), 685-727. MR2077591

[6] H. P. McKean, Selberg's trace formula as applied to a compact Riemann surface, Comm. Pure Appl. Math. 25, (1972), 225-246. MR0473166 (57:12843a)

[7] F. J. Murray and J. von Neumann, On rings of operators, Annals of Math. 37 (1936), 116-229. MR.1503275

[8] M. Pimsner and D. Voiculescu, Imbedding the irrational rotation $C^{*}$-algebra into an $A F$ algebra, J. Operator Theory 4 (1980), 201-210. MR0595412 (82d:46086)

[9] A. Polishchuk and A. Schwarz, Categories of holomorphic vector bundles on noncommutative two-tori, Commun. Math. Phys. 236 (2003), 135-159. MR1977884 (2004k:58011)

[10] N. Ramachandran, Private communication.

[11] M. A. Rieffel, $C^{*}$-algebras associated with irrational rotations, Pacific J. of Math. 93 (1981), 415-429. MR0623572 (83b:46087) 
[12] M. Rørdam, F. Larsen and N. Laustsen, An introduction to $K$-theory for $C^{*}$-algebras, LMS Student Texts, 49, Cambridge University Press, 2000. MR.1783408 (2001g:46001)

[13] P. Schmutz Schaller, Geometry of Riemann surfaces based on closed geodesics, Bull. Amer. Math. Soc. 35 (1998), 193-214. MR1609636 (99b:11098)

[14] J. H. Silverman, The Arithmetic of Elliptic Curves, Springer, 1986. MR0817210 (87g:11070)

[15] Y. Soibelman, Quantum tori, mirror symmetry and deformation theory, Lett. Math. Phys. 56 (2001), 99-125. MR 1854130(2004c:58019)

[16] Y. Soibelman and V. Vologodsky, Non-commutative compactifications and elliptic curves, Int. Math. Res. Not. 28 (2003), 1549-1569. MR.1976601 (2004i:14017)

[17] S. Wolpert, The length spectra as moduli for compact Riemann surfaces, Annals of Math. 109 (1979), 323-351. MR0528966 (80j:58067)

Department of Mathematics, University of Calgary, 2500 University Drive N.W., Calgary, Canada T2N 1N4

E-mail address: nikolaev@math.ucalgary.ca 